\title{
Theoretical investigation of energy levels and transition data for $\mathbf{P} \|^{\star}$
}

\author{
P. Rynkun ${ }^{1}$, L. Radžiūtè ${ }^{1}$, G. Gaigalas ${ }^{1}$, and P. Jönsson ${ }^{2}$ \\ 1 Institute of Theoretical Physics and Astronomy, Vilnius University, Sauletekio av. 3, 10222 Vilnius, Lithuania \\ e-mail: pavel.rynkunatfai.vu.lt \\ 2 Group for Materials Science and Applied Mathematics, Malmö University, 20506 Malmö, Sweden
}

Received 21 November 2018 / Accepted 2 January 2019

\begin{abstract}
Aims. The main goal of this paper is to present accurate and extensive transition data for the P II ion. These data are useful in various astrophysical applications.

Methods. The multiconfiguration Dirac-Hartree-Fock (MCDHF) and relativistic configuration interaction (RCI) methods, which are implemented in the general-purpose relativistic atomic structure package GRASP2K, were used in the present work. In the RCI calculations the transverse-photon (Breit) interaction, the vacuum polarization, and the self-energy corrections were included.

Results. Energy spectra are presented for 48 even states of the $3 s^{2} 3 p^{2}, 3 s^{2} 3 p\{4 p, 4 f, 5 p, 5 f, 6 p\}, 3 s 3 p^{2} 3 d$ configurations, and for 58 odd states of the $3 s 3 p^{3}, 3 s^{2} 3 p\{3 d, 4 s, 4 d, 5 s, 5 d, 6 s\}$ configurations in the P II ion. Electric dipole (E1) transition data are computed between these states along with the corresponding lifetimes. The average uncertainty of the computed transition energies is between five and ten times smaller than the uncertainties from previous calculations. The computed lifetimes for the $3 s^{2} 3 p 4 s^{3} P^{o}$ states are within the error bars of the most current experimental values.
\end{abstract}

Key words. atomic data - radiative transfer

\section{Introduction}

P II is the dominant ion of the ionized phosphorus elements in the neutral interstellar medium. Singly ionized phosphorus has one of the strongest transitions at $1153 \AA\left(3 \mathrm{~s}^{2} 3 \mathrm{p}^{2}{ }^{3} \mathrm{P}-\right.$ $\left.3 \mathrm{~s}^{2} 3 \mathrm{p} 4 \mathrm{~s}^{3} \mathrm{P}^{\mathrm{o}}\right)$ which is typically used to derive phosphorus abundances (Federman et al. 2007). Accurate transition data of P II are useful in astrophysical environments; they are important for the determination of column densities and velocity structure of the interstellar and intergalactic matter (Tayal 2003) .

Hibbert (1988) used configuration interaction as implemented in the CIV3 code to compute energy spectra and probabilities of electric dipole transitions. Tayal (2003) used the multiconfiguration Hartree-Fock (MCHF) method including relativistic corrections through the Breit-Pauli Hamiltonian to calculate energy spectra and transition data for allowed and intercombination lines. Fischer et al. (2006) computed energy levels of the $3 s^{2} 3 p^{2}, 3 s 3 p^{3}, 3 s^{2} 3 p 3 d, 3 s^{2} 3 p 4 s$, and $3 \mathrm{~s}^{2} 3 \mathrm{p} 4 \mathrm{p}$ configurations and transition data using MCHF with the Breit-Pauli approximation for including relativistic corrections. El-Maaref et al. (2012) calculated energy levels, and transition data of silicon-like ions P II, S III, Cl IV, Ar V, and K VI using CIV3. Cashman et al. (2017) compiled the atomic data that are of interest for astrophysics, focusing on the transitions of those ions that have been observed in the Milky Way interstellar medium and/or other galaxies.

Miller et al. (1971) presented the absolute transition probabilities of P I and P II lines measured using the shock-tube tech-

\footnotetext{
* Table 5 is only available at the CDS via anonymous ftp to cdsarc.u-strasbg.fr (130.79.128.5) or via http://cdsarc. u-strasbg.fr/viz-bin/qcat?]/A+A/622/A167
}

nique. Svendenius et al. (1983) measured lines of the P II spectrum in the wavelength region 500-12000 ̊. Federman et al. (2007) presented beam-foil measurements of lifetimes, oscillator strengths, and branching fractions for the multiplet $\left(3 \mathrm{~s}^{2} 3 \mathrm{p}^{2}{ }^{3} \mathrm{P}-\right.$ $3 \mathrm{~s}^{2} 3 \mathrm{p} 4 \mathrm{~s}^{3} \mathrm{P}^{\mathrm{o}}$ ) transitions at $1154 \AA$.

In this work energy spectrum calculations were performed for 48 even states of the $3 s^{2} 3 p^{2}, 3 s^{2} 3 p 4 p, 3 s^{2} 3 p 4 f, 3 s^{2} 3 p 5 p$, $3 s^{2} 3 p 5 f, 3 s^{2} 3 p 6 p$, and $3 s 3 p^{2} 3 d$ configurations, and for 58 odd states of the $3 s^{2} 3 p^{3}, 3 s^{2} 3 p 3 d, 3 s^{2} 3 p 4 s, 3 s^{2} 3 p 4 d, 3 s^{2} 3 p 5 s$, $3 s^{2} 3 p 5 d$, and $3 s^{2} 3 p 6 s$ configurations in P II. Furthermore, electric dipole (E1) transition data were computed between these states.

The calculations were done using multiconfiguration DiracHartree-Fock (MCDHF) and relativistic configuration interaction (RCI) methods (Grant 2007; Fischer et al. 2016), which are implemented in the general-purpose relativistic atomic structure package GRASP2K (Jönsson et al. 2013). In the RCI calculations the transverse-photon (Breit) interaction, the vacuum polarization, and the self-energy corrections were included.

\section{Methods}

\subsection{Computational procedure}

The MCDHF method used in the present work is based on the Dirac-Coulomb Hamiltonian

$H_{\mathrm{DC}}=\sum_{i=1}^{N}\left(c \boldsymbol{\alpha}_{i} \cdot \boldsymbol{p}_{i}+\left(\beta_{i}-1\right) c^{2}+V_{i}^{N}\right)+\sum_{i>j}^{N} \frac{1}{r_{i j}}$,

where $V^{N}$ is the monopole part of the electron-nucleus Coulomb interaction, $\alpha$ and $\beta$ are the $4 \times 4$ Dirac matrices, and $c$ is the speed 
Table 1. Summary of active space construction.

\begin{tabular}{|c|c|c|c|}
\hline \multicolumn{2}{|c|}{ MR set } & \multicolumn{2}{|c|}{$N_{\mathrm{CSFs}}$} \\
\hline Even & Odd & Even & Odd \\
\hline \multicolumn{4}{|c|}{ RCI } \\
\hline $\begin{array}{c}3 s^{2} 3 p^{2}, 3 s^{2} 3 p 4 p, 3 s^{2} 3 p 4 f \\
3 s^{2} 3 p 5 p, 3 s^{2} 3 p 5 f, 3 s^{2} 3 p 6 p \\
3 p^{4}, 3 p^{3} 4 p, 3 p^{3} 4 f, 3 p^{3} 5 p \\
3 p^{3} 5 f, 3 p^{3} 6 p\end{array}$ & $\begin{array}{c}3 \mathrm{~s} 3 \mathrm{p}^{3}, 3 \mathrm{~s}^{2} 3 \mathrm{p} 3 \mathrm{~d}, 3 \mathrm{~s}^{2} 3 \mathrm{p} 4 \mathrm{~s} \\
3 \mathrm{~s}^{2} 3 \mathrm{p} 4 \mathrm{~d}, 3 \mathrm{~s}^{2} 3 \mathrm{p} 5 \mathrm{~s}, 3 \mathrm{~s}^{2} 3 \mathrm{p} 5 \mathrm{~d} \\
3 \mathrm{~s}^{2} 3 \mathrm{p} 6 \mathrm{~s}, 3 \mathrm{~s}^{2} 3 \mathrm{p} 6 \mathrm{~d}, 3 \mathrm{~s}^{2} 3 \mathrm{p} 7 \mathrm{~s} \\
3 \mathrm{p}^{3} 3 \mathrm{~d}, 3 \mathrm{p}^{3} 4 \mathrm{~s}, 3 \mathrm{p}^{3} 4 \mathrm{~d}, 3 \mathrm{p}^{3} 5 \mathrm{~s} \\
3 \mathrm{p}^{3} 5 \mathrm{~d}, 3 \mathrm{p}^{3} 6 \mathrm{~s}, 3 \mathrm{p}^{3} 6 \mathrm{~d}, 3 \mathrm{p}^{3} 7 \mathrm{~s}\end{array}$ & 799548 & 787578 \\
\hline \multicolumn{4}{|c|}{ RCI (CV) additionally included configurations } \\
\hline $\begin{array}{c}3 \mathrm{~s} 3 \mathrm{p}^{2} 3 \mathrm{~d}, 3 \mathrm{~s}^{2} 3 \mathrm{~d}^{2} \\
3 \mathrm{~s}^{2} 3 \mathrm{~d} 7 \mathrm{~d}, 3 \mathrm{~s} 3 \mathrm{p}^{2} 7 \mathrm{~d}\end{array}$ & $3 \mathrm{~s} 3 \mathrm{p}^{2} 4 \mathrm{p}, 3 \mathrm{p}^{3} 7 \mathrm{~d}, 3 \mathrm{~s}^{2} 3 \mathrm{p} 7 \mathrm{~d}$ & 5954032 & 4815663 \\
\hline
\end{tabular}

of light in atomic units. The atomic state functions (ASFs) were obtained as linear combinations of symmetry adapted configuration state functions (CSFs)

$\Psi(\gamma P J M)=\sum_{i=1}^{N_{\mathrm{CSFs}}} c_{i} \Phi\left(\gamma_{i} P J M\right)$.

Here $J$ and $M$ are the angular quantum numbers and $P$ is parity. $\gamma_{i}$ denotes other appropriate labeling of the configuration state function $i$, for example orbital occupancy and coupling scheme. Normally the label $\gamma$ of the atomic state function is the same as the label of the dominating CSF. The CSFs are built from products of one-electron Dirac orbitals. Based on a weighted energy average of several states, the so-called extended optimal level (EOL) scheme (Dyall et al. 1989), both the radial parts of the Dirac orbitals, and the expansion coefficients were optimized to self-consistency in the relativistic self-consistent field procedure.

In subsequent RCI calculations the transverse photon interaction (Breit interaction),

$$
\begin{aligned}
H_{\text {Breit }}= & -\sum_{i<j}^{N}\left[\alpha_{i} \cdot \alpha_{j} \frac{\cos \left(\omega_{i j} r_{i j} / c\right)}{r_{i j}}\right. \\
& \left.+\left(\boldsymbol{\alpha}_{i} \cdot \nabla_{i}\right)\left(\alpha_{j} \cdot \nabla_{j}\right) \frac{\cos \left(\omega_{i j} r_{i j} / c\right)-1}{\omega_{i j}^{2} r_{i j} / c^{2}}\right],
\end{aligned}
$$

was included in the Hamiltonian. The photon frequencies $\omega_{i j}$, used for calculating the matrix elements of the transverse photon interaction, were taken as the difference of the diagonal Lagrange multipliers associated with the Dirac orbitals (McKenzie et al. 1980). In the RCI calculation the leading quantum electrodynamics corrections (QED), self-interaction and vacuum polarization, were also included.

In the present calculations, the ASFs were obtained as expansions over $j j$-coupled CSFs. To provide the $L S J$ labeling system, the ASFs were transformed from a $j j$-coupled CSF basis into an $L S J$-coupled CSF basis using the method provided by Gaigalas et al. (2003, 2017).

\subsection{Computation of transition parameters}

The transition data (transition probabilities, oscillator strengths) between two states $\gamma^{\prime} P^{\prime} J^{\prime} M^{\prime}$ and $\gamma P J M$ can be expressed in terms of the transition moment, which is defined as

$$
\begin{aligned}
& \left\langle\Psi(\gamma P J)\|\mathbf{T}\| \Psi\left(\gamma^{\prime} P^{\prime} J^{\prime}\right)\right\rangle= \\
& \sum_{j, k} c_{j} c_{k}^{\prime}\left\langle\Phi\left(\gamma_{j} P J\right)\|\mathbf{T}\| \Phi\left(\gamma_{k}^{\prime} P^{\prime} J^{\prime}\right)\right\rangle,
\end{aligned}
$$

where $\mathbf{T}$ is the transition operator. For electric dipole and quadrupole (E1 and E2) transitions there are two forms of the transition operator: the length (Babushkin) and velocity (Coulomb) forms, which for the exact solutions of the Dirac-equation give the same value of the transition moment (Grant 1974). The quantity $d T$, characterizing the uncertainty of the computed transition rates, is defined as

$d T=\frac{\left|A_{1}-A_{\mathrm{v}}\right|}{\max \left(A_{1}, A_{\mathrm{v}}\right)}$

where $A_{1}$ and $A_{\mathrm{v}}$ are transition rates in length and velocity forms.

The calculation of the transition moment breaks down to the task of summing up reduced matrix elements between different CSFs.

\section{Scheme of calculations}

As a starting point, MCDHF calculations were performed in the extended optimal level scheme for the weighted average of the even and odd parity states simultaneously. The ASFs were constructed using the multireference-single-double (MRSD) method (Fischer et al. 2016). The MR sets for the even and odd parities are presented in the Table 1, which also displays the number of CSFs in the final even and odd state expansions distributed over the different $J$ symmetries.

The CSF expansions were obtained by allowing SD substitutions from the configurations in the MR to active orbital sets $\{12 \mathrm{~s}, 11 \mathrm{p}, 11 \mathrm{~d}, 10 \mathrm{f}, 9 \mathrm{~g}, 7 \mathrm{~h}, 7 \mathrm{i}\}$. Only CSFs that have nonzero matrix elements with the CSFs belonging to the configurations in the MR were retained. No substitutions were allowed from the $1 \mathrm{~s}, 2 \mathrm{~s}, 2 \mathrm{p}$ shells, which defines an inactive closed core. The MCDHF calculations were followed by RCI calculations, done separately for even and odd states. At the last step, MR was extended and core-valence (CV) correlation (the single substitutions from $2 p$ shell was allowed) was included in the RCI calculations. Single substitutions from $2 \mathrm{p}$ shell were allowed to active orbital sets $\{9 \mathrm{~s}, 8 \mathrm{p}, 8 \mathrm{~d}, 7 \mathrm{f}, 6 \mathrm{~g}\}$.

\section{Results}

In the present work, energy spectra are presented for the 106 (48 even, and 58 odd) lowest states in P II.

To evaluate the accuracy, the obtained transition energies are compared with results from the NIST (Kramida et al. 2018) database, and other theoretical computations (Fig. 1). The figure 


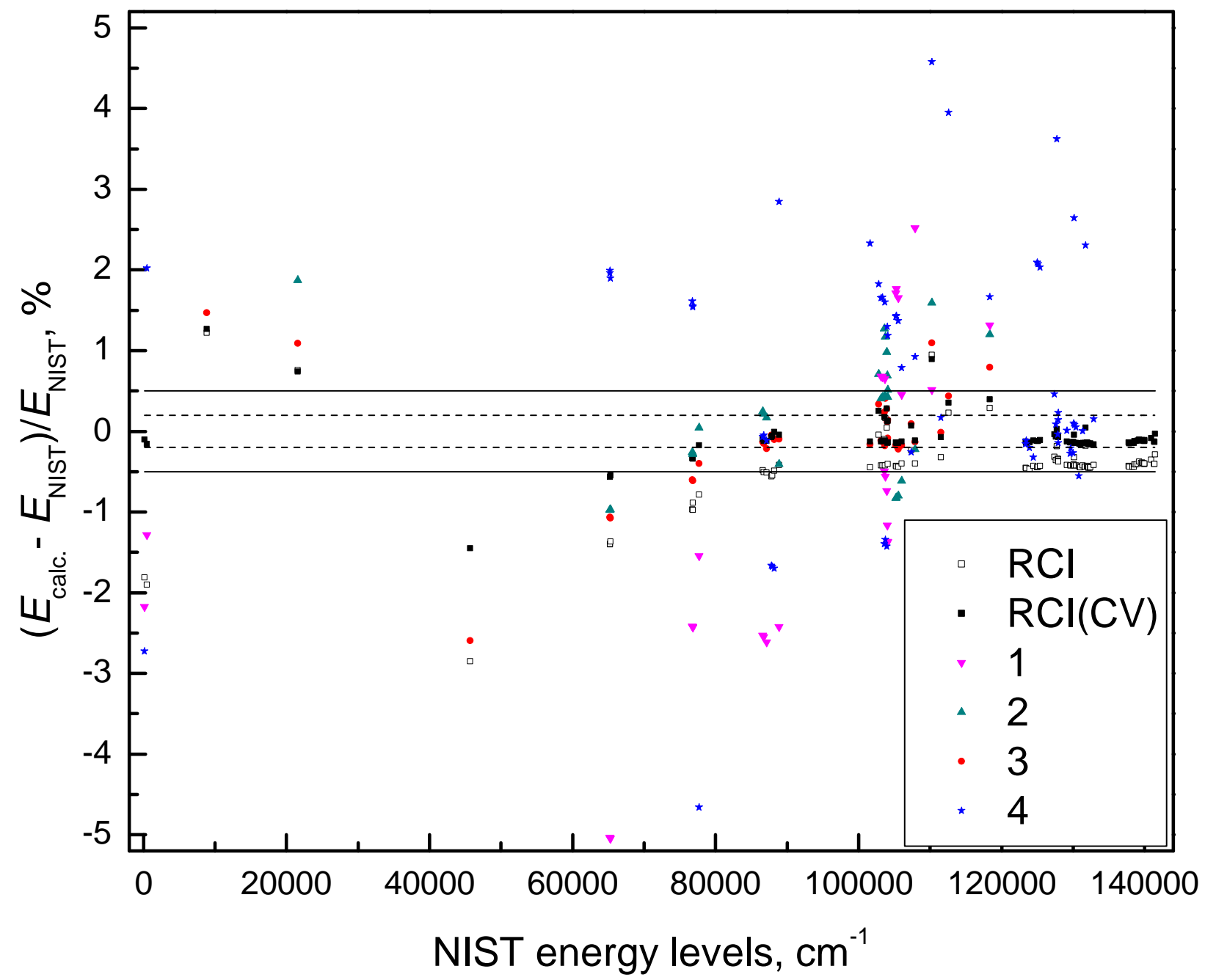

Fig. 1. Comparison of computed energy levels in present work and other theoretical results with data from NIST database. The dashed and solid lines indicate the $0.2 \%$ and $0.5 \%$ deviations, respectively. (1) Hibbert (1988); (2) Tayal (2003); (3) Fischer et al. (2006); (4) El-Maaref et al. (2012).

Table 2. Comparison of computed energy levels in present work and other theoretical results with data from NIST database.

\begin{tabular}{ccc}
\hline \hline Av. accuracy (in \%) & No. of levels in Ref. & Ref. \\
\hline 0.19 & 97 & RCI (CV) \\
2.95 & 31 & 1 \\
1.65 & 32 & 2 \\
1.08 & 40 & 3 \\
1.27 & 63 & 4
\end{tabular}

References. (1) Hibbert (1988); (2) Tayal (2003); (3) Fischer et al. (2006); (4) El-Maaref et al. (2012).

also shows the contributions from core-valence electron correlations and extensions of the MR sets in the present calculations. The final result (RCI (CV)) for the energy spectra agrees very well with NIST. The disagreement is up to $0.15 \%$, except for a few less-excited states where it reaches $1.5 \%$. The averaged uncertainty of computed energy spectra comparing with NIST data is $0.19 \%$. Comparing with other calculations it is seen from Fig. 1 that the results of Fischer et al. (2006) also agree well with the NIST, but the authors calculated less energy levels (41 levels). In Fischer et al. (2006) the largest disagreement is by about $13 \%$ for $3 \mathrm{~s}^{2} 3 \mathrm{p}^{2}\left({ }_{2}^{3} \mathrm{P}\right){ }^{3} \mathrm{P}_{1,2}$ states (these points were not included
Table 3. Comparison of computed lifetimes (in ns) for $3 s^{2} 3 p 4 s^{3} P^{o}$ states of P II ion.

\begin{tabular}{lllc}
\hline \hline$J=0$ & $J=1$ & $J=2$ & Ref. \\
\hline $0.802(0.26)$ & $0.795(0.35)$ & $0.786(0.35)$ & RCI (CV) \\
0.82 & 0.81 & 0.80 & 1 \\
0.784 & 0.778 & 0.772 & 2 \\
0.796 & 0.789 & 0.776 & 3 \\
0.785 & 0.782 & 0.775 & 4 \\
& & 0.80 & 5 \\
$0.79 \pm 0.10$ & $0.79 \pm 0.06$ & $0.84 \pm 0.07$ & 6 (Exp.) \\
$0.85 \pm 0.11$ & $0.85 \pm 0.11$ & $0.85 \pm 0.11$ & 7 (Exp.) \\
$1.3 \pm 0.5$ & $1.3 \pm 0.5$ & $1.3 \pm 0.5$ & 8 (Exp.) \\
\hline
\end{tabular}

Notes. Lifetimes of present calculations are given in length form. The estimated uncertainty on the lifetime is given as a percentage in parentheses.

References. (1) Hibbert (1988); (2) Tayal (2003); (3) Fischer et al. (2006); (4) El-Maaref et al. (2012); (5) Brage et al. (1993); (6) Federman et al. (2007); (7) Livingston et al. (1975); (8) Smith (1978).

in the Fig. 1). In Table 2 a summary of previous calculations is presented: namely, the number of computed energy levels (No. of levels in Ref.) and the average percentage difference between 


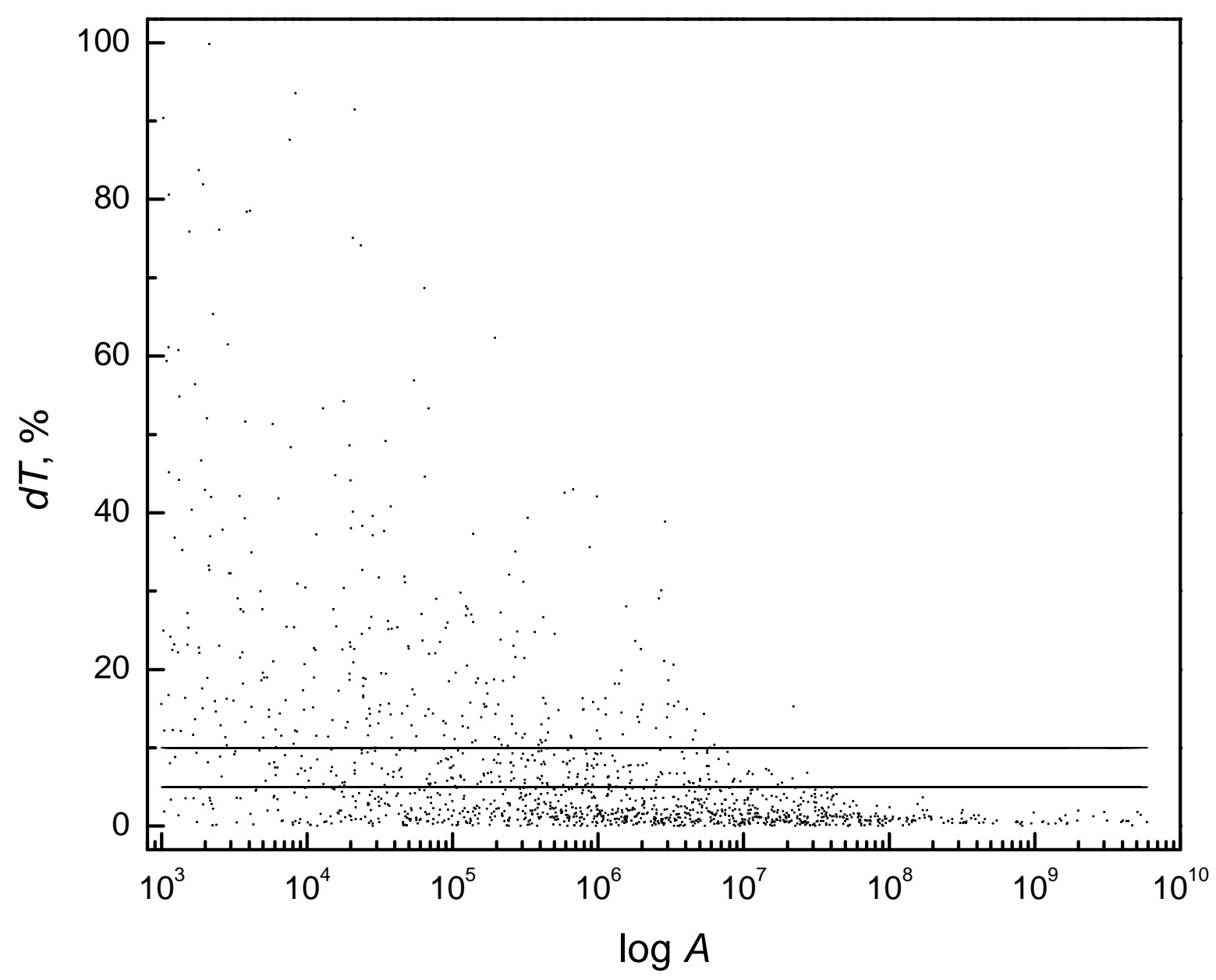

Fig. 2. Scatterplot of $d T$ : the relative difference between the transition rates in length and velocity form vs. the transition rate $A$ for P II. The solid lines indicate the $5 \%$ and $10 \%$ deviations.

Table 4. Comparison of wavelengths and oscillator strengths for the $3 \mathrm{~s}^{2} 3 p^{2}\left({ }_{2}^{3} P\right){ }^{3} P \rightarrow 3 \mathrm{~s}^{2} 3 p^{2} P 4 s^{3} P^{o}$ transitions in P II.

\begin{tabular}{llllllc}
\hline \hline \multicolumn{7}{c}{$J_{i} \rightarrow J_{f}$} \\
$1 \rightarrow 2$ & $0 \rightarrow 1$ & $2 \rightarrow 2$ & $1 \rightarrow 1$ & $1 \rightarrow 0$ & $2 \rightarrow 1$ & Ref. \\
\hline \multicolumn{7}{c}{$f \times 10^{-2}$} \\
\hline $10.66(0.4)$ & $24.97(0.4)$ & $19.00(0.3)$ & $6.19(0.4)$ & $8.36(0.3)$ & $6.27(0.3)$ & RCI (CV) \\
10.4 & 24.4 & 18.6 & 6.1 & 8.1 & 6.2 & 1 \\
10.8 & 25.1 & 19.2 & 6.20 & 8.48 & 6.30 & 2 \\
10.82 & 25.30 & 19.26 & 6.26 & 8.42 & 6.34 & 3 \\
7.8 & 19.0 & 14.0 & 4.7 & 6.2 & 4.7 & 4 \\
$10.5 \pm 1.1$ & $27.2 \pm 2.9$ & $17.4 \pm 1.6$ & $6.4 \pm 0.6$ & $8.5 \pm 1.1$ & $5.9 \pm 0.6$ & 5 (Exp.) \\
\hline \multicolumn{7}{c}{$\lambda($ in $\AA)$} \\
1151.34 & 1154.25 & 1155.38 & 1156.45 & 1158.13 & 1160.53 & RCI (CV) \\
1152.18 & 1154.59 & 1155.68 & 1156.51 & 1158.26 & 1160.04 & 3 \\
1170.41 & 1172.64 & 1174.83 & 1174.88 & 1177.12 & 1179.32 & 4 \\
1149.958 & 1152.818 & 1153.995 & 1155.014 & 1156.970 & 1159.086 & 5 (Exp.) \\
\hline
\end{tabular}

Notes. The oscillator strengths in the RCI column are given in the length gauge. The estimated uncertainty on the oscillator strengths is given as a percentage in parentheses.

References. (1) Hibbert (1988); (2) Tayal (2003); (3) Fischer et al. (2006); (4) El-Maaref et al. (2012); (5) Federman et al. (2007).

NIST and the different methods for the states covered by these methods (Av. accuracy). Final results (RCI (CV)) of energy spectra and lifetimes in length and velocity forms obtained from E1 transitions are displayed in Table A.1. In the present work states of $3 s 3 p^{2} 3 d$ and $3 s^{2} 3 p 6 p$ configurations are presented for the first time.

Lifetimes for $3 \mathrm{~s}^{2} 3 \mathrm{p} 4 \mathrm{~s}{ }^{3} \mathrm{P}^{\mathrm{o}}$ states are compared with experiment and other theoretical calculations in Table 3. Computed 
lifetimes are within the uncertainties of experimental measurement.

Transition data such as wavelengths, weighted oscillator strengths, transition rates of E1 transitions and the accuracy indicator $d T$ are given in Table 5, available at the CDS. Generally, the uncertainty of transition data is small for the stronger transitions. To display this, a scatterplot of $d T$ vs. the transition rate $A$ for computed E1 transitions (with $A>1000 \mathrm{~s}^{-1}$ ) is given in Fig. 2. For most of the strongest transitions, $d T$ is well below $2 \%$. The mean $d T$ for all presented transitions is $7.83 \%$. Table 4 displays the comparison of the theoretical and experimental results of wavelengths and oscillator strengths for the $3 \mathrm{~s}^{2} 3 \mathrm{p}^{2}\left({ }_{2}^{3} \mathrm{P}\right){ }^{3} \mathrm{P} \rightarrow$ $3 \mathrm{~s}^{2} 3 \mathrm{p}{ }^{2} \mathrm{P} 4 \mathrm{~s}{ }^{3} \mathrm{P}^{\mathrm{o}}$ transition. From the table we see that there is very good agreement between wavelengths and oscillator strengths computed in this work and the experimental values (Federman et al. 2007).

\section{Conclusions}

In the present work energy spectra are computed for the 106 lowest states in P II using MCDHF and RCI methods. The mean uncertainty of calculated energy levels comparing with NIST data is $0.19 \%$. The states of $3 \mathrm{~s}^{2} \mathrm{p}^{2} 3 \mathrm{~d}$ and $3 \mathrm{~s}^{2} 3 \mathrm{p} 6 \mathrm{p}$ configurations are presented for the first time.

Transition data for E1 transitions between computed states are presented. For most of the strongest transitions, $d T$ is well below $2 \%$. Lifetimes obtained from E1 transitions are also presented.
Acknowledgements. This research was funded by a grant (No. S-LJB- 18-1) from the Research Council of Lithuania.

\section{References}

Brage, T., Merkelis, G., \& Fischer, C. F. 1993, Phys. Lett. A, 174, 111 Cashman, F. H., Kulkarni, V. P., Kisielius, R., Ferland, G. J., \& Bogdanovich, P. 2017, ApJS, 230, 8

Dyall, K., Grant, I., Johnson, C., Parpia, F., \& Plummer, E. 1989, Comput. Phys. Commun., 55, 425

El-Maaref, A. A., Uosif, M., Allam, S., \& El-Sherbini, T. 2012, At. Data Nucl. Data Tab., 98, 589

Federman, S. R., Brown, M., Torok, S., et al. 2007, ApJ, 660, 919

Fischer, C. F., Tachiev, G., \& Irimia, A. 2006, At. Data Nucl. Data Tab., 92, 607

Fischer, C. F., Godefroid, M., Brage, T., Jönsson, P., \& Gaigalas, G. 2016, J. Phys. B At. Mol. Opt. Phys., 49, 182004

Gaigalas, G., Zalandauskas, T., \& Rudzikas, Z. 2003, At. Data Nucl. Data Tab., 84, 99

Gaigalas, G., Fischer, C., Rynkun, P., \& Jönsson, P. 2017, Atoms, 5, 6

Grant, I. P. 1974, J. Phys. B At. Mol. Opt. Phys., 7, 1458

Grant, I. P. 2007, Relativistic Quantum Theory of Atoms and Molecules (New York: Springer)

Hibbert, A. 1988, Phys. Scr., 38, 37

Jönsson, P., Gaigalas, G., Bieroń, J., Fischer, C. F., \& Grant, I. 2013, Comput. Phys. Commun., 184, 2197

Kramida, A., Ralchenko, Yu., Reader, J., \& NIST ASD Team 2018, NIST Atomic Spectra Database (ver. 5.5.6), https://physics.nist.gov/asd, National Institute of Standards and Technology, Gaithersburg, MD

Livingston, A. E., Kernahan, J. A., Irwin, D. J. G., \& Pinnington, E. H. 1975 Phys. Scr., 12, 223

McKenzie, B., Grant, I., \& Norrington, P. 1980, Comput. Phys. Commun., 21, 233

Miller, M. H., Roig, R. A., \& Bengtson, R. D. 1971, Phys. Rev. A, 4, 1709

Smith, W. H. 1978, Phys. Scr., 17, 513

Svendenius, N., Magnusson, C. E., \& Zetterberg, P. O. 1983, Phys. Scr., 27, 339

Tayal, S. S. 2003, ApJS, 146, 459 


\section{Appendix A: Computed energy levels and lifetimes for the P II ion}

Table A.1. Computed energy levels (in $\mathrm{cm}^{-1}$ ) and lifetimes (in s) in length and velocity gauges for the P II ion.

\begin{tabular}{|c|c|c|c|c|c|}
\hline No. & Label & $\mathrm{RCI}(\mathrm{CV})$ & NIST & $\tau_{l}$ & $\tau_{v}$ \\
\hline 1 & $3 s^{2} 3 p^{2}\left({ }_{2}^{3} P\right)^{3} P_{0}$ & 0 & 0 & & \\
\hline 2 & $3 s^{2} 3 p^{2}\left({ }_{2}^{3} P\right)^{3} P_{1}$ & 164 & 165 & & \\
\hline 3 & $3 s^{2} 3 p^{2}\left({ }_{2}^{3} P\right)^{3} P_{2}$ & 468 & 469 & & \\
\hline 4 & $3 s^{2} 3 p^{2}\left({ }_{2}^{1} D\right){ }^{1} D_{2}$ & 8994 & 8882 & & \\
\hline 5 & $3 s^{2} 3 p^{2}\left({ }_{0}^{1} S\right){ }^{1} S_{0}$ & 21735 & 21576 & & \\
\hline 6 & $3 s 3 p^{3}\left({ }_{3}^{4} S\right){ }^{5} S_{2}^{o}$ & 45035 & 45697 & $1.78 \mathrm{E}-04$ & $1.50 \mathrm{E}-04$ \\
\hline 7 & $3 s 3 p^{3}\left({ }_{3}^{2} D\right){ }^{3} D_{1}^{o}$ & 64881 & 65251 & $9.13 \mathrm{E}-08$ & 8.61E-08 \\
\hline 8 & $3 s 3 p^{3}\left({ }_{3}^{2} D\right)^{3} D_{2}^{o}$ & 64903 & 65272 & $9.23 \mathrm{E}-08$ & 8.68E-08 \\
\hline 9 & $3 s 3 p^{3}\left({ }_{3}^{2} D\right)^{3} D_{3}^{o}$ & 64947 & 65307 & $9.34 \mathrm{E}-08$ & $8.71 \mathrm{E}-08$ \\
\hline 10 & $3 s 3 p^{3}\left({ }_{1}^{2} P\right)^{3} P_{2}^{o}$ & 76504 & 76764 & $1.33 \mathrm{E}-08$ & $1.29 \mathrm{E}-08$ \\
\hline 11 & $3 s 3 p^{3}\left({ }_{1}^{2} P\right)^{3} P_{1}^{o}$ & 76552 & 76812 & $1.23 \mathrm{E}-08$ & $1.19 \mathrm{E}-08$ \\
\hline 12 & $3 s 3 p^{3}\left({ }_{1}^{2} P\right)^{3} P_{0}^{o}$ & 76596 & 76823 & $1.21 \mathrm{E}-08$ & $1.18 \mathrm{E}-08$ \\
\hline 13 & $3 s^{2} 3 p^{2} P 3 d^{1} D_{2}^{o}$ & 77572 & 77710 & $2.05 \mathrm{E}-07$ & $1.88 \mathrm{E}-07$ \\
\hline 14 & $3 s^{2} 3 p^{2} P 4 s^{3} P_{0}^{o}$ & 86510 & 86598 & $8.02 \mathrm{E}-10$ & $7.99 \mathrm{E}-10$ \\
\hline 15 & $3 s^{2} 3 p^{2} P 4 s^{3} P_{1}^{o}$ & 86636 & 86744 & $7.95 \mathrm{E}-10$ & $7.92 \mathrm{E}-10$ \\
\hline 16 & $3 s^{2} 3 p^{2} P 4 s^{3} P_{2}^{o}$ & 87019 & 87125 & $7.86 \mathrm{E}-10$ & $7.84 \mathrm{E}-10$ \\
\hline 17 & $3 s^{2} 3 p^{2} P 3 d^{3} F_{2}^{o}$ & 87741 & 87804 & $3.20 \mathrm{E}-06$ & $3.18 \mathrm{E}-06$ \\
\hline 18 & $3 s^{2} 3 p^{2} P 3 d^{3} F_{3}^{o}$ & 87918 & 87967 & $1.48 \mathrm{E}-06$ & $1.51 \mathrm{E}-06$ \\
\hline 19 & $3 s^{2} 3 p^{2} P 3 d^{3} F_{4}^{o}$ & 88182 & 88192 & & \\
\hline 20 & $3 s^{2} 3 p^{2} P 4 s^{1} P_{1}^{o}$ & 88851 & 88893 & $6.65 \mathrm{E}-10$ & $6.63 \mathrm{E}-10$ \\
\hline 21 & $3 s^{2} 3 p^{2} P 4 p^{1} P_{1}$ & 101508 & 101636 & $1.18 \mathrm{E}-08$ & $1.17 \mathrm{E}-08$ \\
\hline 22 & $3 s^{2} 3 p^{2} P 4 p^{3} D_{1}$ & 103035 & 103166 & $1.00 \mathrm{E}-08$ & 9.97E-09 \\
\hline 23 & $3 s^{2} 3 p^{2} P 3 d^{1} P_{1}^{o}$ & 103059 & 102798 & $4.69 \mathrm{E}-10$ & $4.67 \mathrm{E}-10$ \\
\hline 24 & $3 s^{2} 3 p^{2} P 4 p^{3} D_{2}$ & 103211 & 103339 & $9.95 \mathrm{E}-09$ & $9.88 \mathrm{E}-09$ \\
\hline 25 & $3 s^{2} 3 p^{2} P 4 p^{3} D_{3}$ & 103547 & 103668 & $9.91 \mathrm{E}-09$ & 9.83E-09 \\
\hline 26 & $3 s^{2} 3 p^{2} P 3 d^{3} P_{2}^{o}$ & 103809 & 103630 & $2.15 \mathrm{E}-10$ & $2.14 \mathrm{E}-10$ \\
\hline 27 & $3 s^{2} 3 p^{2} P 3 d^{3} D_{1}^{o}$ & 103911 & 104054 & $1.98 \mathrm{E}-10$ & $1.97 \mathrm{E}-10$ \\
\hline 28 & $3 s^{2} 3 p^{2} P 3 d^{3} D_{3}^{o}$ & 104174 & 104050 & $1.69 \mathrm{E}-10$ & $1.69 \mathrm{E}-10$ \\
\hline 29 & $3 s^{2} 3 p^{2} P 3 d^{3} P_{1}^{o}$ & 104204 & 103756 & $2.12 \mathrm{E}-10$ & $2.10 \mathrm{E}-10$ \\
\hline 30 & $3 s^{2} 3 p^{2} P 3 d^{3} P_{0}^{o}$ & 104231 & 103940 & $2.53 \mathrm{E}-10$ & $2.51 \mathrm{E}-10$ \\
\hline 31 & $3 s^{2} 3 p^{2} P 3 d^{3} D_{2}^{o}$ & 104234 & 104102 & $1.93 \mathrm{E}-10$ & $1.92 \mathrm{E}-10$ \\
\hline 32 & $3 s^{2} 3 p^{2} P 4 p^{3} P_{0}^{2}$ & 105075 & 105224 & 6.67E-09 & $6.66 \mathrm{E}-09$ \\
\hline 33 & $3 s^{2} 3 p^{2} P 4 p^{3} P_{1}$ & 105148 & 105302 & $6.72 \mathrm{E}-09$ & $6.71 \mathrm{E}-09$ \\
\hline 34 & $3 s^{2} 3 p^{2} P 4 p^{3} P_{2}$ & 105396 & 105550 & $6.66 \mathrm{E}-09$ & $6.65 \mathrm{E}-09$ \\
\hline 35 & $3 s^{2} 3 p^{2} P 4 p^{3} S_{1}$ & 105864 & 106001 & 7.53E-09 & $7.52 \mathrm{E}-09$ \\
\hline 36 & $3 s^{2} 3 p^{2} P 3 d^{1} F_{3}^{o}$ & 107436 & 107360 & $2.13 \mathrm{E}-10$ & $2.13 \mathrm{E}-10$ \\
\hline 37 & $3 s^{2} 3 p^{2} P 4 p^{1} D_{2}$ & 107800 & 107923 & 8.65E-09 & 8.59E-09 \\
\hline 38 & $3 s 3 p^{3}\left({ }_{3}^{4} S\right){ }^{3} S_{1}^{o}$ & 111237 & 110255 & $1.10 \mathrm{E}-10$ & $1.08 \mathrm{E}-10$ \\
\hline 39 & $3 s^{2} 3 p^{2} P 4 p^{1} S_{0}$ & 111427 & 111508 & 7.92E-09 & 7.84E-09 \\
\hline 40 & $3 s 3 p^{3}\left({ }_{3}^{2} D\right){ }^{1} D_{2}^{o}$ & 113006 & 112607 & $1.85 \mathrm{E}-10$ & $1.83 \mathrm{E}-10$ \\
\hline 41 & $3 s 3 p^{3}\left({ }_{1}^{2} P\right){ }^{1} P_{1}^{o}$ & 118808 & 118342 & $2.20 \mathrm{E}-10$ & $2.17 \mathrm{E}-10$ \\
\hline 42 & $3 s^{2} 3 p^{2} P 5 s^{3} P_{0}^{o}$ & 123183 & 123344 & 2.05E-09 & $2.05 \mathrm{E}-09$ \\
\hline 43 & $3 s^{2} 3 p^{2} P 5 s^{3} P_{1}^{o}$ & 123277 & 123455 & $2.01 \mathrm{E}-09$ & $2.00 \mathrm{E}-09$ \\
\hline 44 & $3 s^{2} 3 p^{2} P 5 s^{3} P_{2}^{o}$ & 123715 & 123891 & $1.97 \mathrm{E}-09$ & $1.97 \mathrm{E}-09$ \\
\hline 45 & $3 s^{2} 3 p^{2} P 5 s^{1} P_{1}^{o}$ & 124286 & 124432 & $1.68 \mathrm{E}-09$ & $1.68 \mathrm{E}-09$ \\
\hline 46 & $3 s^{2} 3 p^{2} P 4 d^{3} F_{2}^{o}$ & 124792 & 124947 & $4.80 \mathrm{E}-09$ & $4.84 \mathrm{E}-09$ \\
\hline 47 & $3 s^{2} 3 p^{2} P 4 d^{3} F_{3}^{o}$ & 124979 & 125129 & 4.88E-09 & 4.93E-09 \\
\hline 48 & $3 s^{2} 3 p^{2} P 4 d^{3} F_{4}^{o}$ & 125254 & 125391 & $5.14 \mathrm{E}-09$ & $5.21 \mathrm{E}-09$ \\
\hline 49 & $3 s^{2} 3 p^{2} P 4 d^{3} P_{2}^{o}$ & 127318 & 127367 & $6.97 \mathrm{E}-10$ & $6.92 \mathrm{E}-10$ \\
\hline 50 & $3 s^{2} 3 p^{2} P 4 d^{3} D_{1}^{o}$ & 127509 & 127599 & $6.62 \mathrm{E}-10$ & $6.59 \mathrm{E}-10$ \\
\hline
\end{tabular}

Notes. Energy levels are given relative to a ground state energy. 
P. Rynkun et al.: Theoretical investigation of energy levels and transition data for P II

Table A.1. continued.

\begin{tabular}{|c|c|c|c|c|c|}
\hline No. & Label & RCI (CV) & NIST & $\tau_{l}$ & $\tau_{v}$ \\
\hline 51 & $3 s^{2} 3 p^{2} P 4 d^{1} D_{2}^{o}$ & 127789 & 127756 & $4.26 \mathrm{E}-10$ & $4.21 \mathrm{E}-10$ \\
\hline 52 & $3 s^{2} 3 p^{2} P 4 d^{3} D_{3}^{o}$ & 127812 & 127888 & $5.40 \mathrm{E}-10$ & $5.38 \mathrm{E}-10$ \\
\hline 53 & $3 s^{2} 3 p^{2} P 4 d^{3} P_{0}^{o}$ & 127828 & 127900 & $1.03 \mathrm{E}-09$ & $1.03 \mathrm{E}-09$ \\
\hline 54 & $3 s^{2} 3 p^{2} P 4 d^{3} P^{o}$ & 127838 & 127934 & $7.60 \mathrm{E}-10$ & $7.56 \mathrm{E}-10$ \\
\hline 55 & $3 s^{2} 3 p^{2} P 4 d^{3} D_{2}^{o}$ & 127867 & 127950 & $6.21 \mathrm{E}-10$ & $6.17 \mathrm{E}-10$ \\
\hline 56 & $3 s^{2} 3 p^{2} P 5 p^{1} P_{1}^{2}$ & 128944 & 129110 & $1.96 \mathrm{E}-08$ & $2.05 \mathrm{E}-08$ \\
\hline 57 & $3 s^{2} 3 p^{2} P 5 p^{3} D_{1}$ & 129390 & 129569 & $2.48 \mathrm{E}-08$ & $2.59 \mathrm{E}-08$ \\
\hline 58 & $3 s^{2} 3 p^{2} P 5 p^{3} D_{2}$ & 129484 & 129665 & 2.61E-08 & 2.73E-08 \\
\hline 59 & $3 s^{2} 3 p^{2} P 5 p^{3} D_{3}$ & 129847 & 130020 & 2.67E-08 & $2.80 \mathrm{E}-08$ \\
\hline 60 & $3 s^{2} 3 p^{2} P 5 p^{3} P_{0}$ & 129872 & 130058 & $1.42 \mathrm{E}-08$ & $1.49 \mathrm{E}-08$ \\
\hline 61 & $3 s^{2} 3 p^{2} P 5 p^{3} P_{1}$ & 129983 & 130173 & $1.52 \mathrm{E}-08$ & $1.58 \mathrm{E}-08$ \\
\hline 62 & $3 s^{2} 3 p^{2} P 4 d^{1} F_{3}^{o}$ & 130081 & 130143 & $5.52 \mathrm{E}-10$ & $5.53 \mathrm{E}-10$ \\
\hline 63 & $3 s^{2} 3 p^{2} P 5 p^{3} P_{2}^{3}$ & 130210 & 130400 & $1.48 \mathrm{E}-08$ & $1.55 \mathrm{E}-08$ \\
\hline 64 & $3 s^{2} 3 p^{2} P 5 p^{3} S_{1}$ & 130603 & 130801 & $2.10 \mathrm{E}-08$ & $2.19 \mathrm{E}-08$ \\
\hline 65 & $3 s^{2} 3 p^{2} P 4 f^{1} F_{3}$ & 130713 & 130913 & 2.93E-09 & 2.98E-09 \\
\hline 66 & $3 s^{2} 3 p^{2} P 4 f^{3} F_{2}$ & 130744 & 130949 & $3.15 \mathrm{E}-09$ & $3.21 \mathrm{E}-09$ \\
\hline 67 & $3 s^{2} 3 p^{2} P 4 f^{3} F_{3}$ & 130791 & 130993 & $3.06 \mathrm{E}-09$ & $3.11 \mathrm{E}-09$ \\
\hline 68 & $3 s^{2} 3 p^{2} P 4 f^{3} F_{4}$ & 130827 & 131025 & $3.16 \mathrm{E}-09$ & $3.22 \mathrm{E}-09$ \\
\hline 69 & $3 s^{2} 3 p^{2} P 5 p{ }^{1} D_{2}$ & 131162 & 131352 & $1.85 \mathrm{E}-08$ & $1.93 \mathrm{E}-08$ \\
\hline 70 & $3 s^{2} 3 p^{2} P 4 f^{3} G_{3}$ & 131431 & 131631 & 3.01E-09 & 3.04E-09 \\
\hline 71 & $3 s^{2} 3 p^{2} P 4 f^{3} G_{4}$ & 131494 & 131689 & $3.22 \mathrm{E}-09$ & $3.27 \mathrm{E}-09$ \\
\hline 72 & $3 s^{2} 3 p^{2} P 4 f^{3} G_{5}$ & 131757 & 131940 & $3.02 \mathrm{E}-09$ & 3.07E-09 \\
\hline 73 & $3 s^{2} 3 p^{2} P 4 d^{1} P_{1}^{o}$ & 131822 & 131763 & $4.46 \mathrm{E}-10$ & $4.41 \mathrm{E}-10$ \\
\hline 74 & $3 s^{2} 3 p^{2} P 4 f^{1} G_{4}$ & 131886 & 132078 & 4.13E-09 & $4.20 \mathrm{E}-09$ \\
\hline 75 & $3 s^{2} 3 p^{2} P 4 f^{3} D_{3}$ & 131922 & 132132 & $3.42 \mathrm{E}-09$ & 3.44E-09 \\
\hline 76 & $3 s^{2} 3 p^{2} P 4 f^{3} D_{2}$ & 131950 & 132163 & 3.81E-09 & 3.84E-09 \\
\hline 77 & $3 s^{2} 3 p^{2} P 4 f^{3} D_{1}$ & 132161 & 132372 & $3.44 \mathrm{E}-09$ & $3.46 \mathrm{E}-09$ \\
\hline 78 & $3 s^{2} 3 p^{2} P 4 f^{1} D_{2}$ & 132192 & 132397 & 3.86E-09 & 3.89E-09 \\
\hline 79 & $3 s^{2} 3 p^{2} P 5 p{ }^{1} S_{0}$ & 132685 & 132901 & $1.91 \mathrm{E}-08$ & $1.94 \mathrm{E}-08$ \\
\hline 80 & $3 s^{2} 3 p^{2} P 6 s^{3} P_{0}^{o}$ & 137568 & 137757 & $4.21 \mathrm{E}-09$ & 4.25E-09 \\
\hline 81 & $3 s^{2} 3 p^{2} P 6 s^{3} P_{1}^{o}$ & 137621 & 137827 & 3.90E-09 & 3.92E-09 \\
\hline 82 & $3 s 3 p^{2}\left({ }_{2}^{3} P\right){ }^{4} P 3 d^{5} F_{1}$ & 137861 & & $1.44 \mathrm{E}-05$ & $1.35 \mathrm{E}-05$ \\
\hline 83 & $3 s 3 p^{2}\left({ }_{2}^{3} P\right){ }^{4} P 3 d^{5} F_{2}$ & 137923 & & $1.42 \mathrm{E}-05$ & $1.42 \mathrm{E}-05$ \\
\hline 84 & $3 s 3 p^{2}\left({ }_{2}^{3} P\right){ }^{4} P 3 d^{5} F_{3}$ & 138027 & & $1.61 \mathrm{E}-05$ & $1.83 \mathrm{E}-05$ \\
\hline 85 & $3 s^{2} 3 p^{2} P 6 s^{3} P_{2}^{o}$ & 138108 & 138309 & 3.98E-09 & 4.00E-09 \\
\hline 86 & $3 s 3 p^{2}\left({ }_{2}^{3} P\right){ }^{4} P 3 d^{5} F_{4}$ & 138171 & & $2.23 \mathrm{E}-05$ & $2.55 \mathrm{E}-05$ \\
\hline 87 & $3 s^{2} 3 p^{2} P 6 s^{1} P_{1}^{o}$ & 138322 & 138522 & 3.08E-09 & 3.07E-09 \\
\hline 88 & $3 s 3 p^{2}\left({ }_{2}^{3} P\right){ }^{4} P 3 d^{5} F_{5}$ & 138381 & & $8.80 \mathrm{E}-05$ & $8.62 \mathrm{E}-05$ \\
\hline 89 & $3 s^{2} 3 p^{2} P 5 d^{3} F_{2}^{o}$ & 138382 & 138552 & 8.18E-09 & 8.34E-09 \\
\hline 90 & $3 s^{2} 3 p^{2} P 5 d^{3} F_{3}^{o}$ & 138575 & 138743 & 8.43E-09 & 8.64E-09 \\
\hline 91 & $3 s^{2} 3 p^{2} P 5 d^{3} F_{4}^{o}$ & 138881 & 139040 & $1.12 \mathrm{E}-08$ & $1.16 \mathrm{E}-08$ \\
\hline 92 & $3 s 3 p^{2}\left({ }_{2}^{3} P\right){ }^{4} P 3 d^{4} P_{2}$ & 138932 & & $1.83 \mathrm{E}-08$ & $1.77 \mathrm{E}-08$ \\
\hline 93 & $3 s^{2} 3 p{ }^{2} P 5 d^{1} D_{2}^{o}$ & 139072 & 139213 & $1.79 \mathrm{E}-09$ & $1.77 \mathrm{E}-09$ \\
\hline 94 & $3 s^{2} 3 p^{2} P 5 d^{3} D_{1}^{o}$ & 139362 & 139526 & $1.63 \mathrm{E}-09$ & $1.62 \mathrm{E}-09$ \\
\hline 95 & $3 s^{2} 3 p^{2} P 5 d^{3} D_{2}^{o}$ & 139469 & 139623 & $1.81 \mathrm{E}-09$ & $1.79 \mathrm{E}-09$ \\
\hline 96 & $3 s^{2} 3 p^{2} P 5 d^{3} D_{3}^{o}$ & 139644 & 139804 & $1.54 \mathrm{E}-09$ & $1.53 \mathrm{E}-09$ \\
\hline 97 & $3 s^{2} 3 p^{2} P 5 d^{3} P_{2}^{o}$ & 139754 & 139924 & $2.02 \mathrm{E}-09$ & $2.01 \mathrm{E}-09$ \\
\hline 98 & $3 s^{2} 3 p^{2} P 5 d^{3} P_{1}^{o}$ & 139786 & 139958 & $2.45 \mathrm{E}-09$ & 2.43E-09 \\
\hline 99 & $3 s^{2} 3 p^{2} P 5 d^{3} P_{0}^{o}$ & 139815 & 139971 & 2.88E-09 & $2.86 \mathrm{E}-09$ \\
\hline 100 & $3 s^{2} 3 p^{2} P 6 p^{3} D_{2}$ & 140655 & & $3.04 \mathrm{E}-08$ & 3.37E-08 \\
\hline 101 & $3 s^{2} 3 p^{2} P 5 d^{1} F_{3}^{o}$ & 140831 & 140950 & 1.18E-09 & $1.18 \mathrm{E}-09$ \\
\hline 102 & $3 s^{2} 3 p^{2} P 6 p^{3} D_{3}$ & 141055 & & 2.14E-08 & $2.28 \mathrm{E}-08$ \\
\hline 103 & $3 s^{2} 3 p^{2} P 5 f^{1} F_{3}$ & 141141 & 141325 & $6.19 \mathrm{E}-09$ & $6.28 \mathrm{E}-09$ \\
\hline 104 & $3 s^{2} 3 p^{2} P 5 f^{3} F_{3}$ & 141172 & 141354 & 7.23E-09 & $7.41 \mathrm{E}-09$ \\
\hline 105 & $3 s^{2} 3 p^{2} P 5 f^{3} F_{4}$ & 141184 & 141370 & $6.58 \mathrm{E}-09$ & $6.74 \mathrm{E}-09$ \\
\hline 106 & $3 s^{2} 3 p^{2} P 5 d^{1} P_{1}^{o}$ & 141442 & 141488 & $1.06 \mathrm{E}-09$ & $1.05 \mathrm{E}-09$ \\
\hline
\end{tabular}

